\title{
1. Marco general de la Hotelería en Cartagena
}

\author{
Dennis Marrugo Torrente
}

\subsection{RESEÑ̃ HISTÓRICA}

Hablar de la industria sin chimeneas en Cartagena de Indias es remontarnos a la época de la colonia y recordar los lugares que utilizaban los conquistadores para hospedarse al llegar a la isla de Calamarí. Las primeras formas de vivienda utilizadas en la conquista fueron los campamentos. Posteriormente, cuando Cartagena de Indias fue fundada, y sus calles trazadas por Juan de Badillo, se comienza a utilizar las chozas o bohíos hechos en palma y bahareque, materiales disponibles para los conquistadores.

En la época colonial, la ciudad contaba con enormes mansiones de hasta tres pisos, cuyos propietarios eran los gobernadores, alcaldes, marqueses y comerciantes establecidos en la ciudad; contaban con gran cantidad de habitaciones para hospedar a familias y/o amigos comerciantes provenientes del interior del nuevo reino de Granada (Santafé de Bogotá, Popayán, etc). Ellos venían en busca de mercancías provenientes de España, en los galeones que llegaban a Cartagena en la llamada "Feria de Galeones".

En la época de la independencia y mientras la ciudad se fue desarrollando, se comenzó con el arriendo de habitaciones en casas familiares y se abrieron las primeras pensiones conocidas como mesones y hosterías, las cuales fueron un sistema de alojamiento debido a la necesidad de hospedaje de las personas procedentes de otros lugares. Posteriormente en la época de la República, estos lugares toman el carácter típicamente familiar, pero luego se fueron comercializando. 
En la ciudad existieron algunas pensiones, como la pensión Diago, ubicada en la calle de la Universidad y la pensión Guena, frente al parque Centenario. Luego aparecieron las posadas, hoy llamadas hospederías, que dieron origen a los primeros hoteles que existieron en la ciudad. Entre éstos podemos mencionar el Hotel Walter American, hoy edificio Ganem; El Hotel Washington, ubicado frente a la Universidad de Cartagena; el Hotel Vélez, frente a la Plaza Fernández de Madrid; el Hotel Coque, frente a la Plaza de la Aduana, hoy Sena; el Hotel Núñez, el Hotel Alamey, el Hotel Virrey en la esquina de la calle de las Carretas y el Hotel Americano entre otros. Más tarde se construyeron hoteles en los sectores del Cabrero y Marbella, entre los que se encontraban el Hotel Mar, Hotel Calamarí, Hotel Bellavista, Hotel Playa y Sol, etc. Dentro del sector amurallado, también funcionó el Hotel Plaza Bolívar.

\subsection{INICIOS DE LA HOTELERÍA EN CARTAGENA}

En 1944, se construye en Cartagena el primer hotel para turismo: el Hotel Caribe, en Bocagrande. En esa época, eran pocos los esfuerzos que se hacían en pro del turismo. El turismo extranjero estaba interesado en centrar sus actividades en la ciudad de Cartagena, pero era imposible por la falta de hoteles. Luego se dio a conocer la ciudad por el mundo, lo que fue un gran impulso para dar pie al surgimiento de la hotelería en Cartagena, y se vislumbran las posibilidades de la ciudad en este sector.

Debido a la nueva necesidad presentada, se empezó la construcción de algunos hoteles, pues se preveía la necesidad creciente de que la ciudad podría atraer gran cantidad de turistas internacionales que la veían como puerto de escala de América del Sur. Debido a que Cartagena era un puerto de grandes posibilidades, generalmente llegaban veraneantes de Europa, Estados Unidos, y Sur América. De allí nacieron el Hotel Playa, Residencias Bocagrande y el Hotel Bahía. Posteriormente surgieron los demás hoteles como el Capilla del Mar, Hotel Barlovento, Apartahotel las Velas, Apartahotel Cartagena Real, antiguo Hotel Don Blas (hoy Hotel Decamerón), Hotel El Dorado, Hotel Playa, Hotel Cartagena Hilton, Hotel Flamingo, Hotel Costa del Sol, y el Hotel Cartagena Plaza; el Hotel Caribe de Cartagena fue el tercer hotel que se construyó en Colombia. 
La industria turística en cualquier país del mundo, está conformada por tres grandes sectores que son el Turismo, el Transporte y el Alojamiento; la carencia de uno de ellos descompensa el sector; es decir, que sin turismo no hay transporte ni alojamiento.

Es así como Cartagena se ha visto en la necesidad de ampliar los servicios de alojamiento debido a la tecnificación de los medios de transporte, que ahora ofrecen más seguridad y comodidad al viajero, lo que trae como consecuencia la gran afluencia de visitantes.

Es por esto que para vender una ciudad en el exterior, se debe contar con las suficientes habitaciones y camas disponibles. Por ello, la construcción y ampliación de hoteles es de gran importancia para incrementar el turismo.

Hace poco, se abrió el Hotel Santa Clara, restaurado; el Hotel Cartagena Estelar, el Claustro de San Francisco Javier, la compra de la cárcel de San Diego para la construcción de un hotel; también se está ampliando el Cartagena Hilton. Un ejemplo claro de este desarrollo es el hotel las Américas Beach Resort. Con estas construcciones la ciudad posee una capacidad ocupacional mayor que responderá de mejor forma al incrementarse el turismo.

\subsection{RASGOS CARACTERÍSTICOS DE LA ACTIVIDAD TURÍSTICA CARTAGENERA}

Las características naturales, históricas y culturales, y la infraestructura para congresos, convenciones y toda clase de eventos, hacen de Cartagena el destino turístico por excelencia a nivel mundial.

La organización de eventos y convenciones se convierten en un punto fuerte de la oferta turística. El turismo de convenciones y congresos reporta a la ciudad mayores beneficios y ha ganado importancia en los últimos años. Normalmente, este tipo de turismo hace uso de centros y salas de convenciones y de toda la logística que ello implica, de los servicios de traducción simultánea, toures locales entre otros. 
Generalmente, este tipo de eventos internacionales trae consigo a personas con una alta capacidad de gasto, irrigando importantes beneficios económicos a todo el sector (restaurantes, hoteles, transporte, etc.). Es por esto que la ciudad se ha convertido en destino turístico por excelencia. Con la inauguración del Centro de Convenciones, en 1982 y la creación de nuevas instalaciones y el arreglo de algunos puntos turísticos, la ciudad se ha posicionado como el lugar con mayores facilidades para la organización de eventos en la costa atlántica.

En lo referente al turismo de cruceros, éste se remonta a finales de la década de los sesenta, con el arribo a la ciudad de pequeñas naves que transportaban a pocos pasajeros. Posteriormente, se aumenta el número de visitantes y de naves, lo que motiva la construcción de un muelle exclusivo para las actividades de cruceros. El auge de visitantes se ve interrumpido a finales de la década por los problemas de orden público en la ciudad y en el país.

Las gestiones adelantadas por las autoridades locales en la recuperación de la imagen de Cartagena en el exterior contribuyeron a la considerable reactivación de los cruceros en la década de los noventa. Así, el volumen de pasajeros se incrementó considerablemente en los últimos años (Ver cuadro No. 1).

\section{Cuadro No.1}

\section{LLEGADA DE PASAJEROS EN EL MUELLE TURÍSTICO DE MANGA}
AÑOS
1989
1990
1991
1992
1993
1994
1995
1996

No. Buques

68

5

28

54

89

76

124

96
No. de pasajeros

49.978

8.958

21.100

40.648

72.849

60.612

103.346

97.911 
AÑOS

1997

1998

1999

2000

2001
No. Buques

106

136

154

148

151
No. de pasajeros

136.061

156.236

158.311

145.312

147.542

Fuente: Promotora de Turismo. Cámara de Comercio

A pesar de la estacionalidad y al poco tiempo que permanecen en la ciudad, el arribo de cruceros le reporta a la ciudad gran cantidad de beneficios económicos, generando ingresos para los operadores turísticos, guías de turismo, joyerías, taxistas, comercio, transporte, etc.

Gráfica No. 4

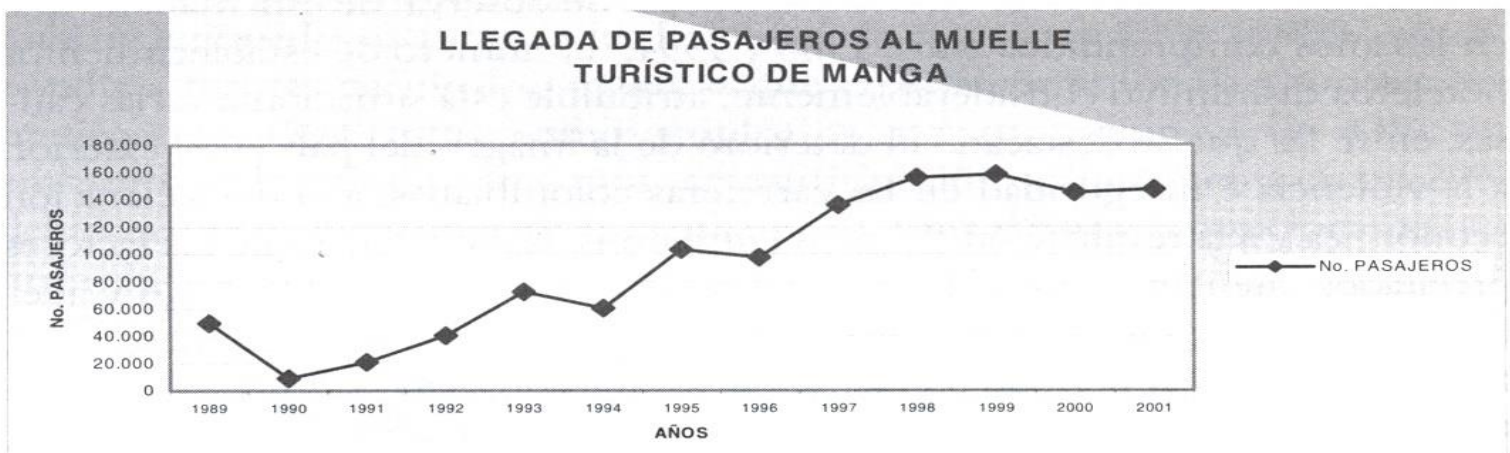

En la serie que se analiza, se puede observar que el número de buques de turismo llegados a Cartagena aumentó de manera significativa en los últimos años. Todo esto debido a la labor de promoción y venta que se ha venido dando de Cartagena en el exterior, lo que ha permitido ver a la ciudad de una manera diferente a la que muestran las noticias.

\subsubsection{COMPORTAMIENTO DEL SECTOR HOTELERO}

Uno de los sectores más afectados por la actual crisis y problemas de orden público que vive el país es el sector turismo; sin embargo, este juega un papel 
estratégico dentro del proceso de reactivación, pues presenta grandes ventajas competitivas y es un importante generador de empleos. A continuación, se describe el comportamiento del sector durante el período de análisis (1991-2001), tomando en consideración algunos indicadores de la demanda turística, representada en los requerimientos de hoteles, agencias de viaje, joyerías, restaurantes, y comercio en general.

Una de las grandes preocupaciones del sector hotelero en la ciudad de Cartagena se refleja en el nivel de ocupación generado. Actualmente, Cartagena contabiliza el $10 \%$ de la instalación hotelera del país. Esta cifra contrasta con el bajo porcentaje que presentaba Cartagena (6.6\%) cuando la Corporación Nacional de Turismo inició la promoción del desarrollo turístico de la Costa. En ese entonces la capacidad hotelera era de 886 habitaciones. Hoy, Cartagena cuenta con 4.864 habitaciones. En el siguiente cuadro, se muestra cuál ha sido el comportamiento del sector en nuestra ciudad. Se observa de esta manera que en los años comprendidos entre 1988 y 1994, el número de establecimientos hoteleros disminuyó considerablemente, atribuible esta situación a varias causas, entre las que se destacan: el deterioro de la imagen del país en el exterior, a la violencia e inseguridad en las carreteras colombianas, a la desaceleración económica y a la revaluación del peso entre otras. Cabe señalar que los factores precitados atentan contra la competitividad de los servicios hoteleros y además motiva gran salida de los turistas nacionales al exterior. Cabe mencionar, además, que en el período siguiente (1995-2001) la actividad presenta síntomas de recuperación al aumentar la oferta de establecimientos y habitaciones, al punto de pasar de 4.044 habitaciones en 1995 a 4864 habitaciones en el 2001

\section{Cuadro No. 2 OFERTA HOTELERA DE CARTAGENA}

$\begin{array}{cccc}\text { Años } & \text { No. Establecimientos } & \text { No. Habitaciones } & \text { No camas } \\ 1985 & 50 & 2.647 & 7.729 \\ 1986 & 51 & 2.702 & 7.512 \\ 1987 & 53 & 2.742 & 7.463 \\ 1988 & 76 & 2.502 & 6.533 \\ 1989 & 59 & 2.824 & 7.494 \\ 1990 & 43 & 3.043 & 8.549\end{array}$




$\begin{array}{cc}\text { Años } & \text { No. Establecimientos } \\ 1991 & 43 \\ 1992 & 42 \\ 1993 & 43 \\ 1994 & 52 \\ 1995 & 70 \\ 1996 & 70 \\ 1997 & 70 \\ 1998 & 102 \\ 1999 & 104 \\ 2000 & 104 \\ 2001 & 104\end{array}$

No. Habitaciones

3.257

3.439

3.632

3.870

4.189

4.444

4.444

4484

4492

4492

4492
No camas

8.963

8.963

9.603

8.447

9.284

9.692

9.689

9.729

9.737

9.737

9.737

Fuente: Promotora de Turismo. Cámara de Comercio.

En términos estadísticos, Cartagena es la segunda ciudad del país (después de Bogotá) que presenta la mayor afluencia de turistas extranjeros. Dentro del total de huéspedes alojados en los hoteles de la ciudad en 1998, el 90\% correspondía a turistas nacionales, mientras que el 10\% correspondía a turistas extranjeros. En 1994, estos últimos ampliaron su participación en un 43\%; sin embargo, en los últimos tres años esta participación se incrementa en un 29\% debido a que se ha recuperado la confianza en venir al país y al posicionamiento de la ciudad en el exterior.

Gráfica No. 2

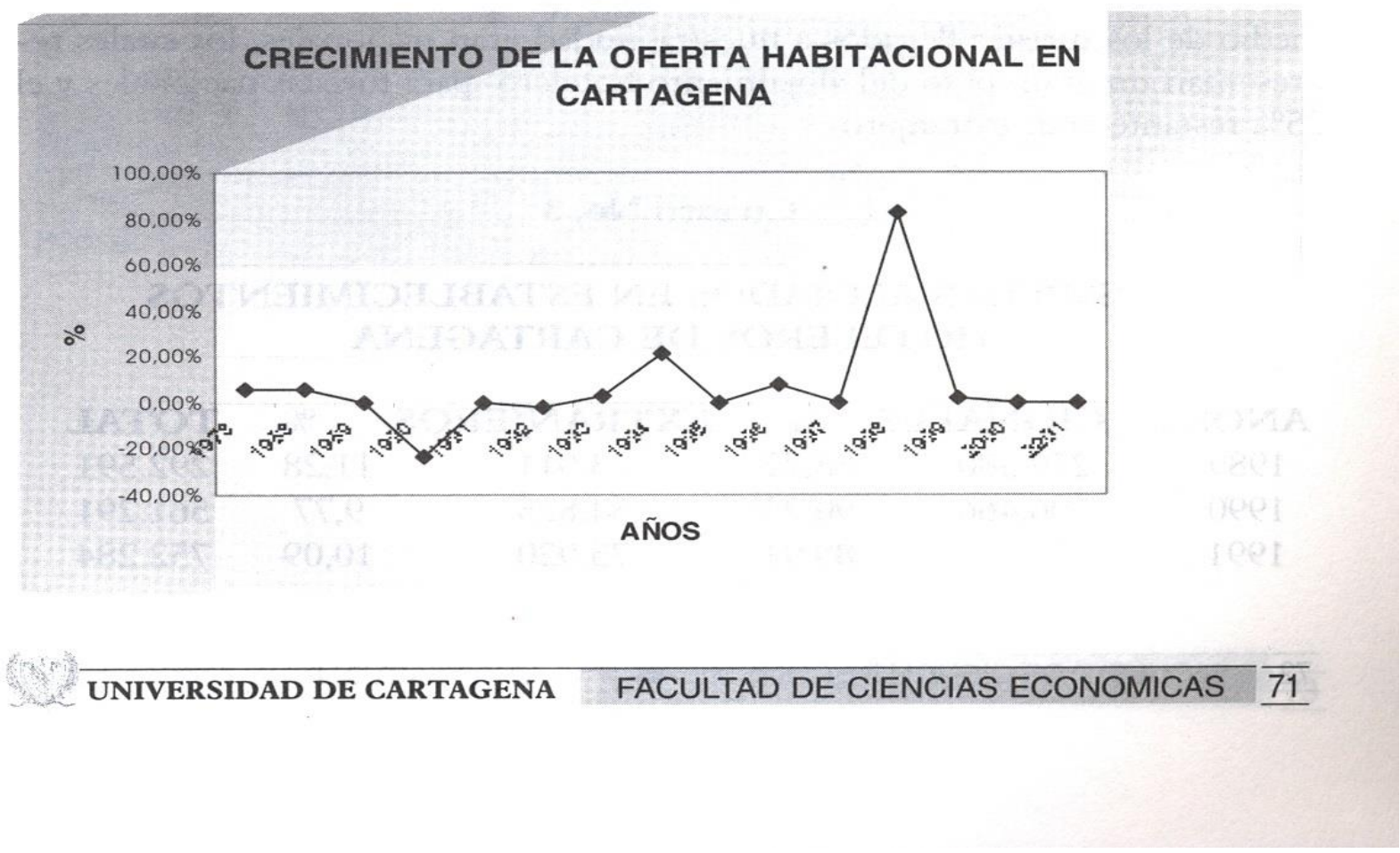


Se puede observar que la oferta hotelera registró un crecimiento negativo en los primeros años de análisis (1986-1993) y que, posteriormente, se observa un repunte, guiado por el posicionamiento de nuestra ciudad a nivel mundial (19952001)

\subsubsection{PROCEDENCIA DE LOS TURISTAS VISITANTES:}

\subsubsection{TURISTAS EXTRANJEROS:}

En lo referente al lugar de procedencia de los turistas que visitaron a Cartagena, puede observarse que la mayor parte de los turistas extranjeros que visitaron nuestra ciudad procedían de los países europeos y de Norteamérica, especialmente en los últimos dos años. El turismo europeo ganó participación entre los años 1994 y 2001, gracias al dinamismo del turismo español e italiano. Lo anterior se debe a las campañas que se están llevando a cabo en otros países, en los que se promociona el turismo nacional, más aún ahora que se habla del turismo ecológico. Estas campañas para promover el turismo se están llevando a cabo no sólo en Europa y Norteamérica, sino también en los países centroamericanos y en los países del Caribe.

\subsubsection{TURISTAS NACIONALES:}

En los trece años de estudio, se observó que aproximadamente el 75\% en promedio de los turistas llegados a nuestra ciudad eran nacionales, los cuales representan un gran peso del alojamiento hotelero para turistas nacionales y el $25 \%$ restante eran extranjeros.

Cuadro No. 3

\section{HUESPEDES ALOJADOS EN ESTABLECIMIENTOS HOTELEROS DE CARTAGENA}

$\begin{array}{cccccc}\text { ANOS } & \text { NACIONALES } & \% & \text { EXTRANJEROS } & \% & \text { TOTAL } \\ 1989 & 259.580 & 88,72 & 33.011 & 11,28 & 292.591 \\ 1990 & 506.466 & 90,23 & 54.825 & 9,77 & 561.291 \\ 1991 & 676.364 & 89,91 & 75.920 & 10,09 & 752.284\end{array}$




$\begin{array}{llllll}1992 & 573.649 & 70,96 & 234.730 & 29,04 & 808.379 \\ 1993 & 466.484 & 62,15 & 284.141 & 37,85 & 750.625 \\ 1994 & 490.341 & 56,66 & 375.038 & 43,34 & 865.379 \\ 1995 & 502.240 & 69,59 & 219.455 & 30,41 & 721.695 \\ 1996 & 492.836 & 79,31 & 128.543 & 20,69 & 621.379 \\ 1997 & 463.567 & 78,86 & 124.283 & 21,14 & 587.850 \\ 1998 & 391.956 & 76,01 & 123.717 & 23,99 & 515.673 \\ 1999 & 379.270 & 70,88 & 155.833 & 29,12 & 535.103 \\ 2000 & 392.520 & 70,73 & 162.450 & 29,27 & 554.970 \\ 2001 & 405.124 & 70,12 & 172.654 & 29,88 & 577.778\end{array}$

Fuente: Promotora de Turismo. Cámara de Comercio

\section{Gráfica No. 3}

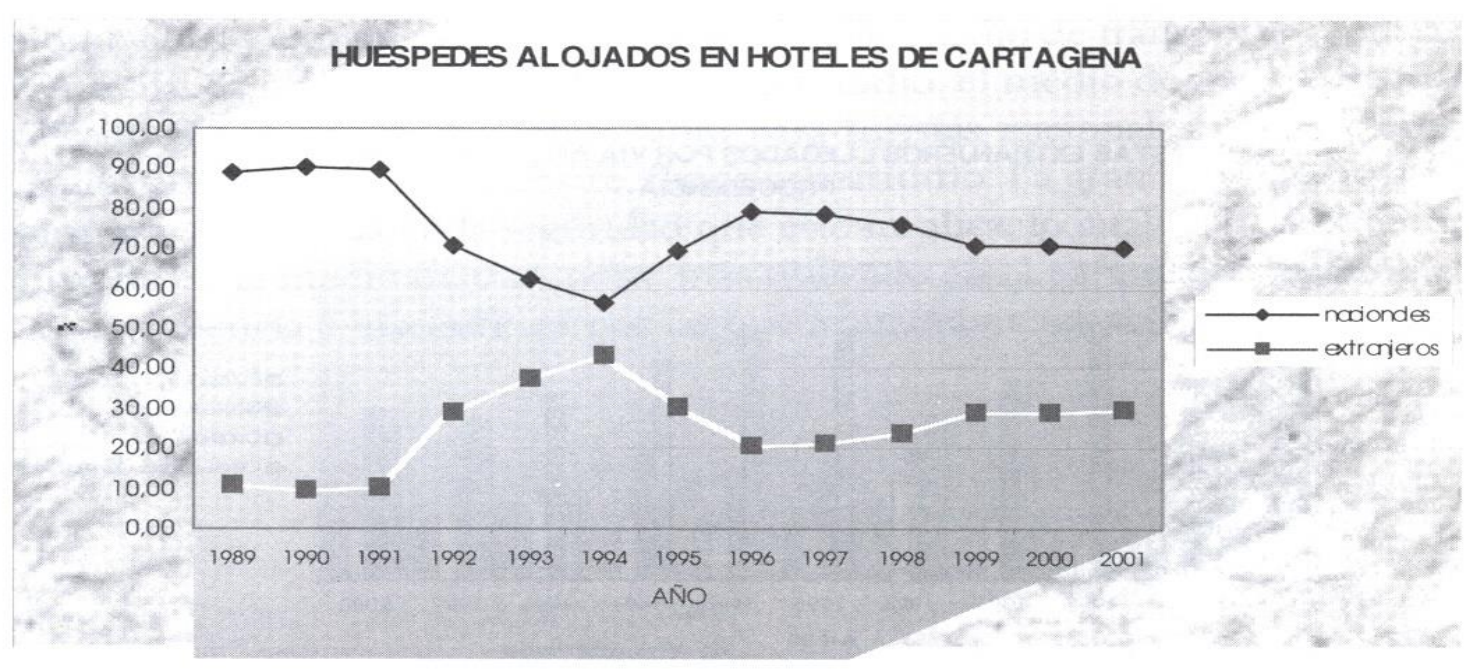


Cuadro No. 4

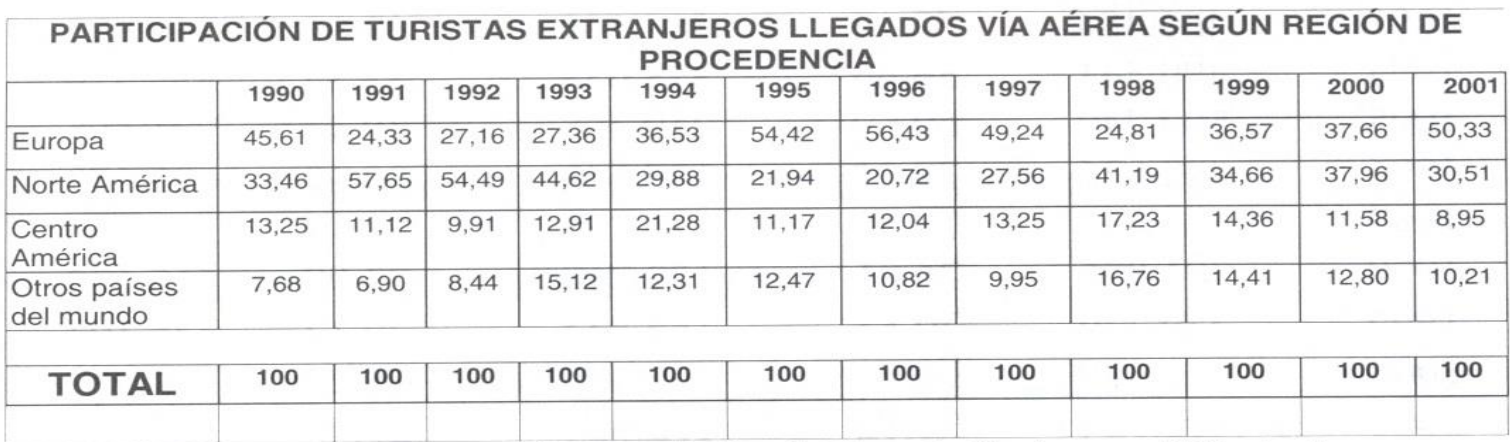

Fuente: Cámara de Comercio. Estadísticas básicas de Cartagena. Cálculo de Cálculo autores. Autores

\section{Gráfica No. 3}

TURISTAS EXTRANUEROS LLEGADOS POR VÍA AÉREA SEGÚN REGIÓN DE PROCEDENCIA

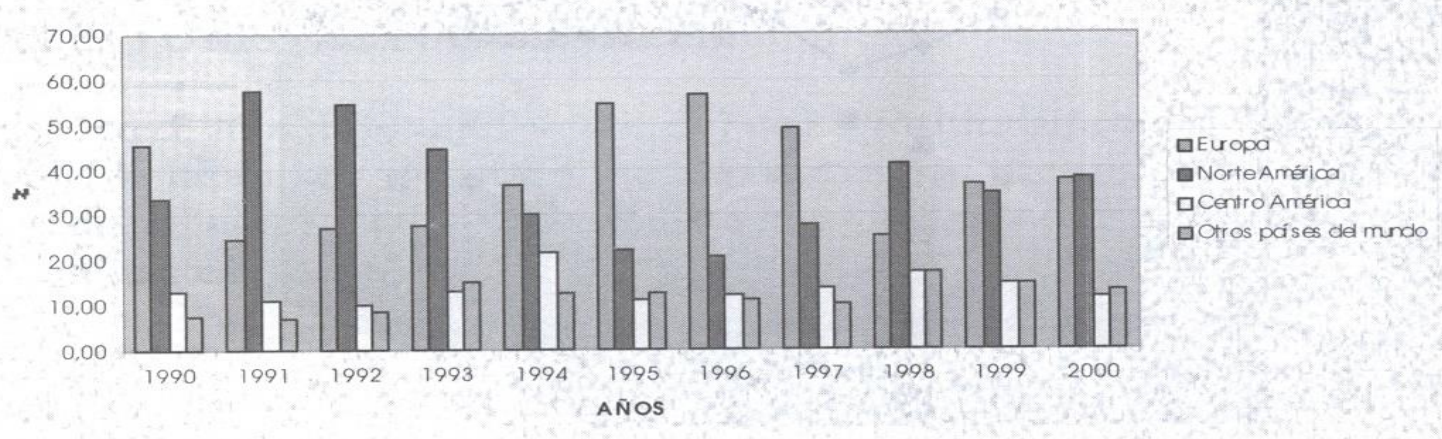


Se observa el importante crecimiento mostrado por el turismo europeo en los años 1994 a 1997; posteriormente, se presenta un decrecimiento en el período de estudio, al pasar de una participación de $49.24 \%$ en 1997 a $24.81 \%$ en 1998 , debido al deterioro de la imagen de nuestro país en el exterior. En los años siguientes, se lanza una campaña masiva a nivel internacional de nuestro país en el exterior, lo que aumenta nuevamente el flujo de turistas europeos a nuestro país.

En lo referente al movimiento de pasajeros por los aeropuertos de la costa atlántica, se observa que los aeropuertos que reportaron mayor movimiento de pasajeros son los aeropuertos de Cartagena, San Andrés y Barranquilla respectivamente, por ser estos en los que se presenta mayor afluencia de turistas en las temporadas altas en la Costa Atlántica. A todo lo anterior, se agrega que éstos son los destinos a los cuales se dirigen los turistas (nacionales y extranjeros) en temporada alta y vacaciones. Se extiende el estudio a los viajeros colombianos y extranjeros entrados al país según medio de transporte y puerto de entrada. Se observa que, para la serie en estudio, el medio de transporte más utilizado fue el transporte aéreo, tanto para turistas nacionales y extranjeros, seguido por el transporte terrestre, fluvial y marítimo. La gran participación del transporte aéreo, está en la seguridad que este implica, lo cual ha hecho que se convierta en el medio de transporte más utilizado en el país, dados los problemas de violencia e inseguridad por las que actualmente atraviesa. 